\title{
Optimal control in heterogeneous domain decomposition methods
}

\author{
V. I. AGOSHKOV*, P. GERVASIO ${ }^{\dagger}$ and A. QUARTERONI ${ }^{\star}$
}

\begin{abstract}
Some new domain decomposition methods (DDM) based on optimal control approach are introduced for the coupling of first- and second-order equations on overlapping subdomains. Several cost functionals and control functions are proposed. Uniqueness and existence results are proved for the coupled problem and the convergence of iterative processes is analyzed.
\end{abstract}

Domain decomposition methods are effective methods of contemporary numerical mathematics for solving boundary value problems [4, 6-10]. To construct these methods, the optimal control approaches can be applied [1, 2, 4, 6-8]. These approaches are used to formulate domain decomposition methods for overlapping and nonoverlapping (disjoint) subdomain decomposition [6, 7], as well as for equations of different orders in each subdomain $[3,4,10]$.

In this paper we analyse some DDM based on the optimal control approaches. We also propose some new algorithms for solving equations of different types in subdomains. Furthermore, we consider the application of our approaches to formulating and studying DDM for systems of equations. The main aim of this paper is to consider DDM for overlapping subdomain decompositions.

The analysis of algorithms considered in the sequel is based on the methodology proposed in [1]. This methodology is primarily based on the theory of operator equations and applications of adjoint operators. The proofs of some statements in this paper are given in [3]. To derive these proofs and analyse the domain decomposition algorithms we use the results of the theory of PDE (the Cauchy problems results, the Poincare problem, problems with oblique derivatives). We also show that the results related to the DDM theory are consequences of the theory of operator equations and PDE.

\footnotetext{
* Institute of Numerical Mathematics, Russian Academy of Sciences, Moscow GSP-1, 119991, Russia

$\dagger$ Department of Mathematics, University of Brescia, 25133 Brescia, Italy

\$MOX, Department of Mathematics, Politecnico di Milano, 20133 Milano, Italy and CMCS-EPFL, CH-1015 Lausanne, Switzerland
}

The work was supported by the Russian Foundation for Basic Research (04-01-00615). 


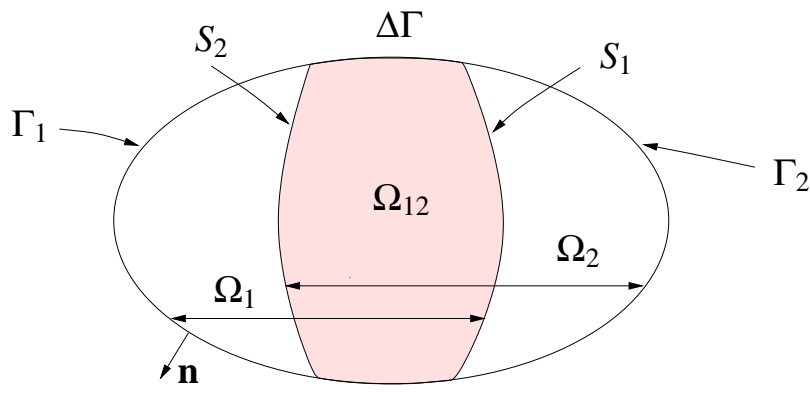

Figure 1. The first possible decomposition.

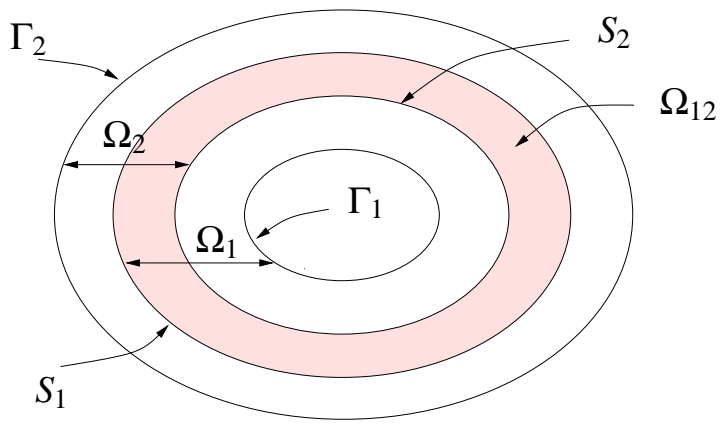

Figure 2. The second possible decomposition.

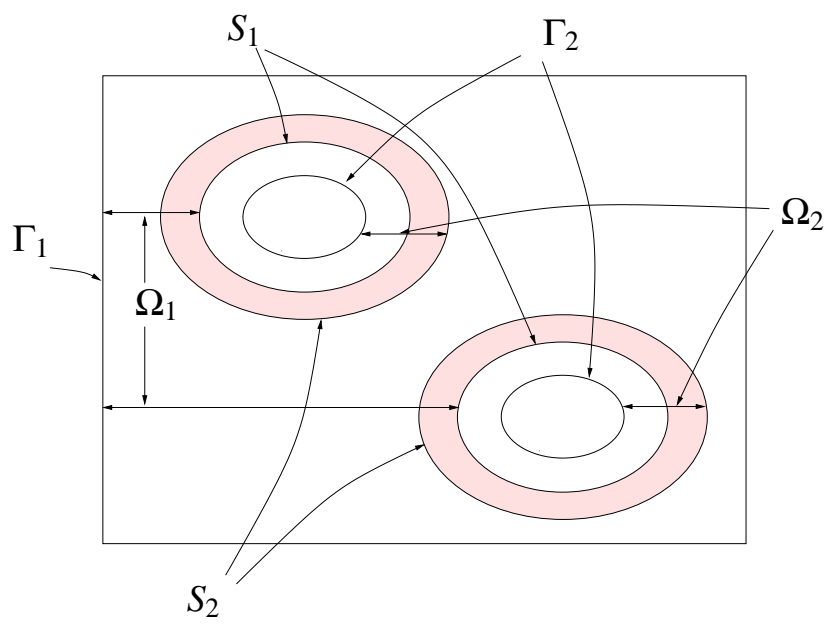

Figure 3. The third possible decomposition. 


\section{NOTATIONS, ASSUMPTIONS AND PRELIMINARY STATEMENTS}

Let $\Omega$ be a two-dimensional domain with the boundary $\Gamma \equiv \partial \Omega$, which is assumed to be Lipschitz continuous and piecewise of class $C^{(2)}, \bar{\Omega}=\Omega \cup \Gamma$. We use the following notations (see Figs. 1-3 for some examples): $\Omega_{1}$ and $\Omega_{2}$ are two subsets of $\Omega$ such that $\bar{\Omega}=\bar{\Omega}_{1} \cup \bar{\Omega}_{2}, \Omega_{1} \cap \Omega_{2} \neq \varnothing, \Omega_{12} \equiv \Omega_{1} \cap \Omega_{2}, \Gamma_{k}=\partial \Omega_{k} \cap \Gamma, S_{k}=$ $\partial \Omega_{k} \backslash \Gamma_{k}, k=1,2$. We consider two situations: when $\Gamma_{1} \cap \Gamma_{2} \neq \varnothing$ (see the case in Fig. 1) and $\Gamma_{1} \cap \Gamma_{2}=\varnothing$ (the cases in Figs. 2, 3). We assume that $\partial \Omega_{1}, \partial \Omega_{2}$ are piecewise of class $C^{(2)}$ and Lipschitz ontinuous.

Let $\mathbf{n}=\left(n_{1}, n_{2}\right)$ be the outward unit normal on $\Gamma, \boldsymbol{\tau}=\left(n_{2},-n_{1}\right)$ the tangent vector; $\mathbf{b}=\left(b_{1}, b_{2}\right)$ be a vector with smooth components. We define: $b_{n}^{k} \equiv$ $\mathbf{b} \cdot \mathbf{n} \equiv \sum_{i=1}^{2} b_{i} n_{i} \quad$ on $\partial \Omega_{k}, b_{n}^{k}=\left(b_{n}^{k}\right)^{+}-\left(b_{n}^{k}\right)^{-},\left(b_{n}^{k}\right)^{+}=\left(\left|b_{n}^{k}\right|+b_{n}^{k}\right) / 2,\left(b_{n}^{k}\right)^{-}=$ $\left(\left|b_{n}^{k}\right|-b_{n}^{k}\right) / 2, k=1,2$. We will use the real spaces $L_{2}(\Omega), L_{2}\left(\Omega_{k}\right), L_{2}(\Gamma), \ldots, L_{2}\left(\Gamma_{k}\right)$, $k=1,2$, as well as the following spaces:

$$
\begin{aligned}
& L_{2}\left(S_{k}^{-}\right):=\left\{u \in L_{2}\left(S_{k}\right):\|u\|_{L_{2}\left(S_{k}^{-}\right)}=\left(\int_{S_{k}}\left(b_{n}^{k}\right)^{-}|u|^{2} \mathrm{~d} \Gamma\right)^{1 / 2}<\infty\right\}, \quad k=1,2 \\
& L_{2}\left(S_{k}^{+}\right):=\left\{u \in L_{2}\left(S_{k}\right):\|u\|_{L_{2}\left(S_{k}^{+}\right)}=\left(\int_{S_{k}}\left(b_{n}^{k}\right)^{+}|u|^{2} \mathrm{~d} \Gamma\right)^{1 / 2}<\infty\right\}, \quad k=1,2 \\
& L_{2}\left(\Gamma_{k}^{-}\right):=\left\{u \in L_{2}\left(\Gamma_{k}\right):\|u\|_{L_{2}\left(\Gamma_{k}^{-}\right)}=\left(\int_{\Gamma_{k}}\left(b_{n}^{k}\right)^{-}|u|^{2} \mathrm{~d} \Gamma\right)^{1 / 2}<\infty\right\}, \quad k=1,2 \\
& L_{2}\left(\Gamma_{k}^{+}\right):=\left\{u \in L_{2}\left(\Gamma_{k}\right):\|u\|_{L_{2}\left(\Gamma_{k}^{+}\right)}=\left(\int_{\Gamma_{k}}\left(b_{n}^{k}\right)^{+}|u|^{2} \mathrm{~d} \Gamma\right)^{1 / 2}<\infty\right\}, \quad k=1,2
\end{aligned}
$$

where $S_{k}^{-}=S_{k} \cap\left\{\left(b_{n}^{k}\right)^{-} \neq 0\right\}, S_{k}^{+}=S_{k} \cap\left\{\left(b_{n}^{k}\right)^{+} \neq 0\right\}, \Gamma_{k}^{-}=\Gamma_{k} \cap\left\{\left(b_{n}^{k}\right)^{-} \neq 0\right\}$, $\Gamma_{k}^{+}=\Gamma_{k} \cap\left\{\left(b_{n}^{k}\right)^{+} \neq 0\right\}$. Let us consider the differential operators

$$
\begin{aligned}
& L_{1} u_{1} \equiv \operatorname{div}\left(\mathbf{b} u_{1}\right)+b_{0} u_{1} \quad \text { in } \Omega_{1} \\
& L_{2} u_{2} \equiv-v \Delta u_{2}+\operatorname{div}\left(\mathbf{b} u_{2}\right)+b_{0} u_{2} \quad \text { in } \Omega_{2}
\end{aligned}
$$

where $v=$ const $>0, \mathbf{b}$ and $b_{0}$ are such that $\left(b_{0}+(\operatorname{divb}) / 2\right) \geqslant \mu_{0}=$ const $>0$ $\forall x \in \bar{\Omega}$. Each operator $L_{k}$ is defined on smooth functions in $\Omega_{k}, k=1,2$. The operator $L_{k}^{(0)}, k=1,2$, is defined by (1.1) on smooth functions which satisfies the homogeneous boundary conditions on $\Gamma_{k}$. We consider the following problem: find $u_{1}, u_{2}, \lambda_{1}, \lambda_{2}$ such that

$$
\begin{gathered}
L_{1} u_{1}=f \text { in } \Omega_{1}, \quad\left(b_{n}^{1}\right)^{-} u_{1}=\left(b_{n}^{1}\right)^{-} g \text { on } \Gamma_{1}, \quad\left(b_{n}^{1}\right)^{-} u_{1}=\left(b_{n}^{1}\right)^{-} \lambda_{1} \text { on } S_{1} \\
L_{2} u_{2}=f \quad \text { in } \Omega_{2}, \quad u_{2}=g \quad \text { on } \Gamma_{2}, \quad u_{2}=\lambda_{2} \quad \text { on } S_{2} \\
u_{1}=u_{2} \text { in } \Omega_{12}
\end{gathered}
$$

where $f$ is a given function defined in $\bar{\Omega}$, while the function $g$ is defined on $\partial \Omega$. Hereafter we assume that all $\mathbf{b}, f_{0}, f, g$ in (1.2) are smooth in $\bar{\Omega}$. 
Problem (1.2) is an 'exact controllability problem' with two 'controls' $\lambda_{1}, \lambda_{2}$. Let us study some uniqueness and existence results for (1.2), which are used later.

If $\left\{u_{1}, u_{2}, \lambda_{1}, \lambda_{2}\right\}$ is a smooth solution of (1.2) we can consider the equation $L_{1} u_{1}=f_{1}$ on $\partial \Omega_{12}$ to find

$$
b_{n} \frac{\partial u_{1}}{\partial n}+b_{\tau} \frac{\partial u_{1}}{\partial \tau}+\mu u_{1}=f \quad \text { on } \partial \Omega_{12}
$$

where $\mu \equiv b_{0}+\operatorname{div} \mathbf{b}, b_{n}=\mathbf{b} \cdot \mathbf{n}, b_{\tau}=\mathbf{b} \cdot \boldsymbol{\tau}$ and $\mathbf{n}$ is the outward unit normal to $\partial \Omega_{12}$.

Let us state the first proposition.

Proposition 1.1 [3]. Problem (1.2) has no solution in the general case, i.e., without introducing specific restriction to the data of the problem.

We introduce the following types of assumptions:

I.

$$
\left\{\begin{array}{l}
\Omega_{12}=\bigcup_{k=1}^{m} \Omega_{12}^{(k)} \quad \Delta \Gamma=\bigcup_{\substack{k=1 \\
m}}^{m} \Delta \Gamma^{(k)}, \quad \Delta \Gamma^{(k)} \subset \partial \Omega_{12}^{(k)}, \quad m<\infty \\
\left.\operatorname{meas}\left(\Delta \Gamma^{(k)}\right)>0, \quad b_{n} \neq 0 \quad \text { on } \Delta \Gamma^{(k)} \quad \text { (see Fig. } 1\right)
\end{array}\right.
$$

where $\Omega_{12}^{(k)}=\Omega_{12} \cap \Omega_{k}$.

II.

$$
\left\{\begin{array}{l}
\Omega_{12} \text { is finite, } \mu=b_{0}+\operatorname{div} \mathbf{b} \geqslant 0 \quad \text { on } \partial \Omega_{12}, \quad \mu \not \equiv 0 \quad \text { on } \partial \Omega_{12} \\
\text { the direction } \mathbf{b} \text { at any point of } \partial \Omega_{12} \text { makes an acute angle } \\
\text { with the outward normal to } \partial \Omega_{12} .
\end{array}\right.
$$

III.

$$
\left\{\begin{array}{l}
\Omega_{12}=\bigcup_{k=1}^{m} \Omega_{12}^{(k)}, \quad b_{n} \neq 0 \quad \text { on } \partial \Omega_{12}, \quad \frac{\mu}{b_{n}}-\frac{1}{2} \frac{\partial}{\partial \tau}\left(\frac{b_{\tau}}{b_{n}}\right)>0 \quad \text { on } \partial \Omega_{12} \\
\text { where } \frac{\partial}{\partial \tau} \text { is the derivative along } \partial \Omega_{12} .
\end{array}\right.
$$

The following proposition is valid.

Proposition 1.2 [3]. If problem (1.2) has a solution and one of the assumptions I-III is valid, this solution is unique.

The assertions of Propositions 1.1, 1.2 will be used in the next section while investigating a domain decomposition algorithm based on optimal control. Note also that analogous assertions can be proved for the case $\Omega \subset \mathbb{R}^{n}, n>2$, and for a system of equations of type (1.2). The results of the Cauchy problems, the problems of the oblique derivative, and the Poincare problem are still useful for proving these assertions. 


\section{ANALYSIS OF DDM WITH TWO CONTROL FUNCTIONS}

Let us consider the weak statement of (1.2): find $\left\{u_{1}, u_{2}, \lambda_{1}, \lambda_{2}\right\}$ such that

$$
\begin{aligned}
& L_{1} u_{1}=f \quad \text { in } \Omega_{1}, \quad\left(b_{n}^{1}\right)^{-} u_{1}=\left(b_{n}^{1}\right)^{-} g \text { on } \Gamma_{1}, \quad\left(b_{n}^{1}\right)^{-} u_{1}=\left(b_{n}^{1}\right)^{-} \lambda_{1} \quad \text { on } S_{1} \\
& L_{2} u_{2}=f \text { in } \Omega_{2}, \quad u_{2}=g \text { on } \Gamma_{2}, u_{2}=\lambda_{2} \text { on } S_{2} \\
& \inf _{\lambda_{1}, \lambda_{2}} J_{0}\left(u_{1}, u_{2}\right)
\end{aligned}
$$

where

$$
J_{0}\left(u_{1}, u_{2}\right)=\frac{1}{2} \int_{\Omega} \chi_{12}\left(u_{1}(\lambda)-u_{2}(\lambda)\right)^{2} \mathrm{~d} \Omega
$$

function $\chi_{12}$ is the characteristic function of $\Omega_{12}$, and $\lambda=\left(\lambda_{1}, \lambda_{2}\right)$ is the vector of 'controls' $\lambda_{1}, \lambda_{2}$.

Consider the following iterative method: for given $\lambda^{0}$, solve

$$
\begin{gathered}
L_{1} u_{1}^{m}=f \text { in } \Omega_{1}, \quad\left(b_{n}^{1}\right)^{-} u_{1}^{m}=\left(b_{n}^{1}\right)^{-} g \quad \text { on } \Gamma_{1}, \quad\left(b_{n}^{1}\right)^{-} u_{1}^{m}=\left(b_{n}^{1}\right)^{-} \lambda_{1}^{m} \text { on } S_{1} \\
L_{2} u_{2}^{m}=f \quad \text { in } \Omega_{2}, \quad u_{2}^{m}=g \quad \text { on } \Gamma_{2}, \quad u_{2}^{m}=\lambda_{2}^{m} \quad \text { on } S_{2} \\
\lambda^{m+1}=\lambda^{m}-\gamma_{m} J_{0}^{\prime}\left(u_{1}^{m}, u_{2}^{m}\right), \quad m=0,1, \ldots
\end{gathered}
$$

where $\left\{\gamma_{m}\right\}$ are suitable relaxation parameters to be chosen according to convergence criteria [1,9-13].

Algorithm (2.2) is in fact a domain decomposition method for solving (1.2). In this section we analyse problem (2.1) and the convergence of the approximate solution obtained by iterative process (2.2).

The variational equations ('optimality conditions') corresponding to (2.1) read as follows:

$$
\begin{gathered}
L_{1} u_{1}=f \text { in } \Omega_{1}, \quad\left(b_{n}^{1}\right)^{-} u_{1}=\left(b_{n}^{1}\right)^{-} g \quad \text { on } \Gamma_{1}, \quad\left(b_{n}^{1}\right)^{-} u_{1}=\left(b_{n}^{1}\right)^{-} \lambda_{1} \text { on } S_{1} \\
L_{2} u_{2}=f \quad \text { in } \Omega_{2}, \quad u_{2}=g \quad \text { on } \Gamma_{2}, \quad u_{2}=\lambda_{2} \quad \text { on } S_{2} \\
L_{1}^{(0) *} q_{1}=\chi_{12}\left(u_{1}-u_{2}\right) \text { in } \Omega_{1}, \quad\left(b_{n}^{1}\right)^{+} q_{1}=0 \text { on } \Gamma_{1}, \quad\left(b_{n}^{1}\right)^{+} q_{1}=0 \text { on } S_{1} \\
L_{2}^{(0) *} q_{2}=\chi_{12}\left(u_{1}-u_{2}\right) \quad \text { in } \Omega_{2}, \quad q_{2}=0 \text { on } \partial \Omega_{2} \\
\left(b_{n}^{1}\right)^{-} q_{1}=0 \text { on } S_{1}, \quad v \frac{\partial q_{2}}{\partial n}=0 \text { on } S_{2}
\end{gathered}
$$


while the iterative process (2.2) can be rewritten as: for a given $\lambda^{0}$

$$
\begin{gathered}
L_{1} u_{1}^{m}=f \text { in } \Omega_{1}, \quad\left(b_{n}^{1}\right)^{-} u_{1}^{m}=\left(b_{n}^{1}\right)^{-} g \text { on } \Gamma_{1}, \quad\left(b_{n}^{1}\right)^{-} u_{1}^{m}=\left(b_{n}^{1}\right)^{-} \lambda_{1}^{m} \text { on } S_{1} \\
L_{2} u_{2}^{m}=f \text { in } \Omega_{2}, \quad u_{2}^{m}=g \text { on } \Gamma_{2}, \quad u_{2}^{m}=\lambda_{2}^{m} \text { on } S_{2} \\
L_{1}^{(0) *} q_{1}^{m}=\chi_{12}\left(u_{1}^{m}-u_{2}^{m}\right) \text { in } \Omega_{1}, \quad\left(b_{n}^{1}\right)^{+} q_{1}^{m}=0 \text { on } \partial \Omega_{1} \\
L_{2}^{(0) *} q_{2}^{m}=\chi_{12}\left(u_{1}^{m}-u_{2}^{m}\right) \text { in } \Omega_{2}, \quad q_{2}^{m}=0 \text { on } \partial \Omega_{2} \\
\left(b_{n}^{1}\right)^{-} \lambda_{1}^{m+1}=\left(b_{n}^{1}\right)^{-} \lambda_{1}^{m}-\gamma_{m}\left(b_{n}^{1}\right)^{-} q_{1}^{m} \text { on } S_{1}, \quad \lambda_{2}^{m+1}=\lambda_{2}^{m}-\gamma_{m} v \frac{\partial q_{2}^{m}}{\partial n} \text { on } S_{2} .
\end{gathered}
$$

Proposition 2.1 [3]. (1) Problem (2.1) (resp. (2.3)) has no solution in the general case.

(2) If problem (2.1) (resp. (2.3)) has a solution, in the general case $\inf _{\lambda} J_{0}\left(u_{1}, u_{2}\right)>$ 0 , i.e., $u_{1} \neq u_{2}$ in $\Omega_{12}$.

(3) If one of the assumptions I-III is satisfied and the problem (2.1) (resp. (2.3)) has a solution, this solution is unique.

(4) If the problem (2.1) (resp. (2.3)) has a unique solution and the iterative process (2.2) (resp. (2.4)) is convergent, generally

$$
\lim _{m \rightarrow \infty}\left\|u_{1}^{m}-u_{2}^{m}\right\|_{L_{2}\left(\Omega_{12}\right)} \geqslant \text { const }>0
$$

i.e., $u_{1}^{m}, u_{2}^{m}$ do not generally concide in $\Omega_{12}$ as $m \rightarrow \infty$.

From Proposition 2.1 we can draw the following conclusion: in order for the property $\lim _{k \rightarrow \infty}\left\|u_{1}^{k}-u_{2}^{k}\right\|=0$ to hold, statement (2.1) has to be modified. One possibility, which consists in introducing a third control (in addition to $\lambda_{4}, \lambda_{2}$ ), will be investigated in the next section.

Remark 2.1. We can study the 'regularized problem' (instead of (2.1)):

$$
\begin{gathered}
L_{1} u_{1}=f \text { in } \Omega_{1}, \quad\left(b_{n}^{1}\right)^{-} u_{1}=\left(b_{n}^{1}\right)^{-} g \text { on } \Gamma_{1}, \quad\left(b_{n}^{1}\right)^{-} u_{1}=\left(b_{n}^{1}\right)^{-} \lambda_{1} \text { on } S_{1} \\
L_{2} u_{2}=f \text { in } \Omega_{2}, \quad u_{2}=g \text { on } \Gamma_{2}, \quad u_{2}=\lambda_{2} \text { on } S_{2} \\
\inf _{\lambda_{1}, \lambda_{2}} \frac{1}{2}\left(\alpha \int_{S_{1}}\left(b_{n}^{1}\right)^{-} \lambda_{1}^{2} \mathrm{~d} \Gamma+\alpha \int_{S_{2}} \lambda_{2}^{2} \mathrm{~d} \Gamma\right)+J_{0}\left(u_{1}, u_{2}\right), \quad \alpha \geqslant 0 .
\end{gathered}
$$

If $\alpha>0$, this problem has the unique solution $u_{k}(\alpha), \lambda_{k}(\alpha), k=1,2$. The associated iterative process (2.2) converges: $u_{k}^{m}(\alpha) \rightarrow u_{k}(\alpha), \lambda_{k}^{m}(\alpha) \rightarrow \lambda_{k}(\alpha)$ for $k=1,2$ as $m \rightarrow \infty$, where $\left\{u_{k}\right\},\left\{\lambda_{k}\right\}$ is the solution of (2.6). However, we cannot prove that $u_{k}(\alpha) \rightarrow u_{k}$ and $\lambda_{k}(\alpha) \rightarrow \lambda_{k}$ for $k=1,2$ as $\alpha \rightarrow 0$. In fact, if problem (1.2) has some solutions, $\left\{u_{k}(\alpha)\right\},\left\{\lambda_{k}(\alpha)\right\}$ converge to the solution with the minimal norm of $\left\{\lambda_{k}\right\}$. However, if problem (1.2) (or (2.1)) has no solution, we cannot expect the convergence of the iterative process in the general case or $\left\{u_{k}(\alpha)\right\},\left\{\lambda_{k}(\alpha)\right\}$ converge to a pseudosolution. 


\section{DDM WITH THREE CONTROL FUNCTIONS}

In this section we propose and analyse a domain decomposition algorithm to solve problem (1.2) making use of three control functions.

Let $\omega$ be a smooth function in $\Omega$ such that $0 \leqslant \omega(x) \leqslant 1$ in $\Omega, \omega=0$ in $\Omega \backslash \Omega_{12}$, $\omega>0$ in $\Omega_{12}$. Let us consider the problem: find $u_{k}, \lambda_{k}, k=1,2, v \in L_{2}\left(\Omega_{12}\right)$ such that

$$
\begin{gathered}
L_{1} u_{1}=f+\omega v \text { in } \Omega_{1}, \quad\left(b_{n}^{1}\right)^{-} u_{1}=\left(b_{n}^{1}\right)^{-} g \text { on } \Gamma_{1}, \quad\left(b_{n}^{1}\right)^{-} u_{1}=\left(b_{n}^{1}\right)^{-} \lambda_{1} \text { on } S_{1} \\
L_{2} u_{2}=f \text { in } \Omega_{2}, \quad u_{2}=g \text { on } \Gamma_{2}, \quad u_{2}=\lambda_{2} \text { on } S_{2} \\
u_{1}=u_{2} \text { in } \Omega_{12} .
\end{gathered}
$$

The optimal control problem associated with (3.1)-(3.3) reads: find $u_{k}=u_{k}(\alpha)$, $\lambda_{k}=\lambda_{k}(\alpha), k=1,2, v=v(\alpha)$ which satisfy (3.1), (3.2) and, moreover,

$$
\inf _{\lambda_{1}, \lambda_{2}, v} J_{\alpha}\left(u_{1}, u_{2}, \lambda_{1}, \lambda_{2}, v\right)
$$

where

$$
\begin{aligned}
J_{\alpha}\left(u_{1}, u_{2}, \lambda_{1}, \lambda_{2}, v\right)= & \frac{1}{2}\left(\alpha \int_{S_{1}}\left(b_{n}^{1}\right)^{-} \lambda_{1}^{2} \mathrm{~d} \Gamma+\alpha \int_{S_{2}} \lambda_{2}^{2} \mathrm{~d} \Gamma\right. \\
& \left.+\alpha \int_{\Omega} \omega v^{2} \mathrm{~d} \Omega+\int_{\Omega} \chi_{12}\left(u_{1}-u_{2}\right)^{2} \mathrm{~d} \Omega\right)
\end{aligned}
$$

and $\alpha=\mathrm{const} \geqslant 0$ is the regularization parameter.

If $\alpha=0,(3.1),(3.2)$ and (3.4) are the weak statement of problem (3.1)-(3.3). In the sequel, we identify $L_{2}\left(\Omega_{12}\right)$ with the subspace $L_{2}^{(0)}\left(\Omega_{k}\right)=\left\{u: u \in L_{2}\left(\Omega_{k}\right)\right.$, $u \equiv 0$ in $\left.\Omega_{k} \backslash \Omega_{12}\right\}$. The multiplication of $u \in L_{2}\left(\Omega_{12}\right)$ by $\chi_{12}$ will be considered as the prolongation by zero of $u$ onto $\Omega_{k} \backslash \Omega_{12}$.

The minimization requirement (3.4) yields the set of optimality equations:

$$
\begin{gathered}
L_{1}^{(0) *} q_{1}=\chi_{12}\left(u_{1}-u_{2}\right) \text { in } \Omega_{1}, \quad\left(b_{n}^{1}\right)^{+} q_{1}=0 \text { on } \partial \Omega_{1} \\
L_{2}^{(0) *} q_{2}=\chi_{12}\left(u_{1}-u_{2}\right) \text { in } \Omega_{2}, \quad q_{2}=0 \text { on } \partial \Omega_{2} \\
\alpha\left(b_{n}^{1}\right)^{-} \lambda_{1}+\left(b_{n}^{1}\right)^{-} q_{1}=0 \text { on } S_{1}, \quad \alpha \lambda_{2}+v \frac{\partial q_{2}}{\partial n}=0 \text { on } S_{2} \\
\alpha \omega v+\omega q_{1}=0 \text { in } \Omega_{1} .
\end{gathered}
$$

We consider the iterative process to solve (3.1), (3.2) and (3.5): for any given 
$\lambda_{1}^{0}, \lambda_{2}^{0}, v^{0}$ for $m \geqslant 1$ we look for $\lambda_{1}^{m+1}, \lambda_{2}^{m+1}, v^{m+1}$ such that

$$
\begin{gathered}
L_{1} u_{1}^{m}=f+\omega v^{m} \text { in } \Omega_{1}, \quad\left(b_{n}^{1}\right)^{-} u_{1}^{m}=\left(b_{n}^{1}\right)^{-} g \text { on } \Gamma_{1} \\
\left(b_{n}^{1}\right)^{-} u_{1}^{m}=\left(b_{n}^{1}\right)^{-} \lambda_{1}^{m} \text { on } S_{1} \\
L_{2} u_{2}^{m}=f \text { in } \Omega_{2}, \quad u_{2}^{m}=g \text { on } \Gamma_{2}, \quad u_{2}^{m}=\lambda_{2}^{m} \text { on } S_{2} \\
L_{1}^{(0) *} q_{1}^{m}=\chi_{12}\left(u_{1}^{m}-u_{2}^{m}\right) \text { in } \Omega_{1}, \quad\left(b_{n}^{1}\right)^{+} q_{1}^{m}=0 \text { on } \partial \Omega_{1} \\
L_{2}^{(0) *} q_{2}^{m}=\chi_{12}\left(u_{1}^{m}-u_{2}^{m}\right) \text { in } \Omega_{2}, \quad q_{2}^{m}=0 \text { on } \partial \Omega_{2} \\
\left(b_{n}^{1}\right)^{-} \lambda_{1}^{m+1}=\left(b_{n}^{1}\right)^{-} \lambda_{1}^{m}-\gamma_{m}\left(\alpha\left(b_{n}^{1}\right)^{-} \lambda_{1}^{m}+\left(b_{n}^{1}\right)^{-} q_{1}^{m}\right) \text { on } S_{1} \\
\lambda_{2}^{m+1}=\lambda_{2}^{m}-\gamma_{m}\left(\alpha \lambda_{2}^{m}+v \frac{\partial q_{2}^{m}}{\partial n}\right) \text { on } S_{2} \\
v^{m+1}=v^{m}-\gamma_{m}\left(\alpha v^{m}+q_{1}^{m}\right) \text { in } \Omega_{12}, m=0,1, \ldots
\end{gathered}
$$

The parameters $\left\{\gamma_{m}\right\}$ have to be chosen to make these equations converge.

Hereafter we use the notion of 'dense solvability' for problem (3.1)-(3.3) (see [5]).

Definition 3.1. We say that problem (3.1)-(3.3) is densely solvable (or the property of dense solvability holds for (3.1)-(3.3)) if for any $q>0$ there are functions $\widetilde{\lambda}_{1}, \widetilde{\lambda}_{2}, \widetilde{v}$ such that the problems

$$
\begin{gathered}
L_{1} \widetilde{u}_{1}=f+\omega \widetilde{v} \text { in } \Omega_{1}, \quad\left(b_{n}^{1}\right)^{-} \widetilde{u}_{1}=\left(b_{n}^{1}\right)^{-} g \text { on } \Gamma_{1}, \quad\left(b_{n}^{1}\right)^{-} \widetilde{u}_{1}=\left(b_{n}^{1}\right)^{-} \widetilde{\lambda}_{1} \text { on } S_{1} \\
L_{2} \widetilde{u}_{2}=f \text { in } \Omega_{2}, \quad \widetilde{u}_{2}=g \text { on } \Gamma_{2}, \quad \widetilde{u}_{2}=\widetilde{\lambda}_{2} \text { on } S_{2}
\end{gathered}
$$

have the solutions $\widetilde{u}_{1}, \widetilde{u}_{2}$ such that

$$
\left\|\widetilde{u}_{1}-\widetilde{u}_{2}\right\|_{L_{2}\left(\Omega_{12}\right)} \leqslant \varepsilon_{1} .
$$

(This is also referred to as a property of 'approximate' controllability for problem (3.1)-(3.3).)

Let us now consider problem (3.1)-(3.3). Should two solutions $\left\{u_{1}^{(1)}, u_{2}^{(1)}, \lambda_{1}^{(1)}\right.$, $\left.\lambda_{2}^{(1)}, v^{(1)}\right\}$ and $\left\{u_{1}^{(2)}, u_{2}^{(2)}, \lambda_{1}^{(2)}, \lambda_{2}^{(2)}, v^{(2)}\right\}$ exist, their difference $u_{1}=u_{1}^{(1)}-u_{1}^{(2)}$, $\ldots, v=v^{(1)}-v^{(2)}$ would satisfy the equations

$$
\begin{gathered}
L_{1} u_{1}=\omega v \text { in } \Omega_{1}, \quad\left(b_{n}^{1}\right)^{-} u_{1}=0 \text { on } \Gamma_{1}, \quad\left(b_{n}^{1}\right)^{-} u_{1}=\left(b_{n}^{1}\right)^{-} \lambda_{1} \text { on } S_{1} \\
L_{2} u_{2}=0 \text { in } \Omega_{2}, \quad u_{2}=0 \text { on } \Gamma_{2}, \quad u_{2}=\lambda_{2} \text { on } S_{2} \\
u_{1}=u_{2}, \text { in } \Omega_{12} .
\end{gathered}
$$

From (3.9) for $u_{1} \equiv u_{2} \equiv u$ in $\Omega_{12}$ we obtain the boundary value problem

$$
\begin{aligned}
& L_{2} u=0 \text { in } \Omega_{12} \\
& L_{1} u=b_{n} \frac{\partial u}{\partial n}+b_{\tau} \frac{\partial u}{\partial \tau}+\mu u=\omega v=0 \text { on } \partial \Omega_{12} .
\end{aligned}
$$


If assumptions (1.4) are valid, we have:

$$
L_{2} u=0 \text { in } \Omega_{12}, \quad u=\frac{\partial u}{\partial n}=0 \text { on } \Delta \Gamma^{(k)}, \quad k=1, \ldots, m
$$

$u=0$ in $\Omega_{12}$ by the uniqueness continuation theorem. Hence, $v=0, \lambda_{k}=0, u_{k}=0$ in $\Omega_{k}, k=1,2$, i.e. the solutions of (3.1)-(3.3) are unique.

The same conclusion holds when assumption (1.5) or (1.6) rather than (1.4) is valid.

The uniqueness result is valid for problem (3.1), (3.2), (3.5) (equivalently (3.1), (3.2), (3.4)) for any $\alpha \geqslant 0$. Let us study the existence of solutions of this problem. If $\alpha>0$, existence and uniqueness are proven by invoking the results of [1].

Now, we consider the case $\alpha=0$. First, we prove the 'dense solvability' of (3.1)-(3.3). For this purpose let us consider the homogeneous adjoint problem corresponding to (3.1)-(3.3): find $q_{1}, q_{2}, w$ such that

$$
\begin{gathered}
L_{1}^{(0) *} q_{1}=\chi_{12} w \text { in } \Omega_{1}, \quad\left(b_{n}^{1}\right)^{+} q_{1}=0 \text { on } \partial \Omega_{1} \\
L_{2}^{(0) *} q_{2}=\chi_{12} w \text { in } \Omega_{2}, \quad q_{2}=0 \text { on } \partial \Omega_{2} \\
\left(b_{n}^{1}\right)^{-} q_{1}=0 \text { on } S_{1}, \quad v \frac{\partial q_{2}}{\partial n}=0 \text { on } S_{2}, \quad \omega q_{1}=0 \text { in } \Omega_{1} .
\end{gathered}
$$

The last relation yields: $q_{1}=0$ in $\Omega_{12}$. Now, using the equations in $\Omega_{1}$ and $\Omega_{2}$, we also conclude that $w=0$ in $\Omega_{12}, q_{k}=0$ in $\Omega_{k}, k=1,2$. So the adjoint problem (3.11) admits only a trivial solution. If we apply the theory of operator equations $[1,5]$, we have the dense solvability of (3.1)-(3.3). Besides, the solutions of (3.1), (3.2), (3.5) for sufficiently small $\alpha>0$ can be chosen as 'regularized approximations' of the solutions of (3.1)-(3.3) such that (see [1]):

$$
\begin{gathered}
L_{1} u_{1}=f+\omega v \text { in } \Omega_{1}, \quad\left(b_{n}^{1}\right)^{-} u_{1}=\left(b_{n}^{1}\right)^{-} g \text { on } \Gamma_{1} \\
L_{2} u_{2}=f \text { in } \Omega_{2}, \quad u_{2}=g \text { on } \Gamma_{2}, \quad b_{2}=\lambda_{2} \text { on } S_{2} \\
J_{0}\left(u_{1}, u_{2}\right)=\frac{1}{2}\left\|\chi_{12}\left(u_{1}-u_{2}\right)\right\|_{L_{2}(\Omega)}^{2} \leqslant \varepsilon_{1}
\end{gathered}
$$

and

$$
\left\|\chi_{12}\left(u_{1}-u_{2}\right)\right\|_{L_{2}(\Omega)}^{2} \rightarrow 0 \text { as } \alpha \rightarrow+0
$$

where $u_{k}=u_{k}(\alpha), \lambda_{k}=\lambda_{k}(\alpha), k=1,2, v=v(\alpha)$ are the solution of (3.1), (3.2), (3.5).

Remark 3.1. Using (3.13) and the results from the theory of PDE, it is easy to obtain some additional convergence results such as

$$
\left\|\mathbf{b} \cdot \nabla q_{1}\right\|_{L_{2}\left(\Omega_{1}\right)}+\left\|q_{1}\right\|_{L_{2}\left(\Omega_{1}\right)}+\left\|q_{2}\right\|_{L_{2}\left(\Omega_{2}\right)} \rightarrow 0, \quad\left\|q_{2}\right\|_{H^{1}\left(\Omega_{2}\right)} \rightarrow 0 \text { as } \alpha \rightarrow+0
$$


and $\left\|q_{2}\right\|_{H^{2}\left(\Omega_{2}\right)} \rightarrow 0$ as $\alpha \rightarrow+0$ if $\Omega_{2}$ is convex or $\partial \Omega_{2}$ is smooth.

Let us formulate the assertion.

Proposition 3.1. For problems (3.1)-(3.3) and (3.1), (3.2), (3.5) the following statements hold true:

(1) Problem (3.1)-(3.3) is densely solvable.

(2) For any $\alpha>0$ problem (3.1), (3.2), (3.5) (equivalently, (3.1), (3.2), (3.4)) has the unique solution $u_{k}=u_{k}(\alpha), \lambda_{k}=\lambda_{k}(\alpha), k=1,2, v=v(\alpha)$ and

$$
\left\|\chi_{12}\left(u_{1}-u_{2}\right)\right\|_{L_{2}(\Omega)} \rightarrow 0 \text { as } \alpha \rightarrow+0
$$

(3) If problem (3.1)-(3.3) has the unique solution $u_{k}^{(0)}, \lambda_{k}^{(0)}, k=1,2, v^{(0)}$,

$$
u_{k}(\alpha) \rightarrow u_{k}^{(0)}, \quad \lambda_{k}(\alpha) \rightarrow \lambda_{k}^{(0)}, \quad k=1,2, \quad v(\alpha) \rightarrow v^{(0)} \text { as } \alpha \rightarrow+0 .
$$

(4) If $\left\{u_{k}^{m}\right\},\left\{\lambda_{k}^{m}\right\}, k=1,2, v^{m}$ are calculated by the convergent iterative process (3.6), for any $\varepsilon_{2}>0$ there are a small $\alpha>0$ and a sufficiently large $m=M \gg$ 1 such that $\left\|\chi_{12}\left(u_{1}^{m}-u_{2}^{m}\right)\right\|_{L_{2}(\Omega)} \leqslant \varepsilon_{2}$, i.e., $\left\{u_{k}^{m}\right\},\left\{\lambda_{k}^{m}\right\}, k=1,2, v^{m}$ can be considered as an approximate solution of (3.1)-(3.3).

(5) If (3.1)-(3.3) has the unique solution $u_{k}^{(0)}, \lambda_{k}^{(0)}, k=1,2, v^{(0)}$,

$$
u_{k}^{m} \rightarrow u_{k}^{(0)}, \quad \lambda_{k}^{m} \rightarrow \lambda_{k}^{(0)}, \quad k=1,2, \quad v^{m} \rightarrow v^{(0)} \text { as } \alpha \rightarrow 0 \text { and } m \rightarrow \infty
$$

and for a sufficiently small $\alpha>0$ and large $m=M \gg 1$ the functions $u_{k}^{m}, \lambda_{k}^{m}, v^{m}$ can be chosen as approximations of $u_{k}^{(0)}, \lambda_{k}^{(0)}, v^{(0)}, k=1,2$.

Statements (1), (2) have been formulated earlier. The proof of (3)-(5) follows from (1), (2), the convergence of (3.6) and the general results of the theory of illposed problems $[1,11,12]$.

Remark 3.2. We call attention to the following point: if $\left\|u_{1}-u_{2}\right\|_{L_{2}\left(\Omega_{12}\right)} \rightarrow 0$, $\alpha \rightarrow+0$ or $\left\|u_{1}^{m}-u_{2}^{m}\right\|_{L_{2}\left(\Omega_{12}\right)} \rightarrow 0$ as $\alpha \rightarrow+0$ and $m \rightarrow \infty$, we do not expect the convergence of both $v(\alpha)$ and $v^{m}(\alpha)$ to zero as $\alpha \rightarrow+0$ and $m \rightarrow \infty$ in the general case (because in this case it can be in contradiction with the results from Propositions 1.1 and 1.2). 


\section{DOMAIN DECOMPOSITION ALGORITHMS WITH 'MIXED-TYPE' CONTROLS}

Now we study domain decomposition algorithms based on optimal control approaches with different types of controls on $S_{1}^{-}, S_{2}^{-}$and in $\Omega_{12}$. We consider the following optimal control problem: find $u_{1}, u_{2}, \lambda_{1}, \lambda_{2}, v$ such that

$$
\begin{gathered}
L_{1} u_{1}=f+\omega v \text { in } \Omega_{1}, \quad\left(b_{n}^{1}\right)^{-} u_{1}=\left(b_{n}^{1}\right)^{-} g \text { on } \Gamma_{1} \\
\left(b_{n}^{1}\right)^{-} u_{1}=\left(b_{n}^{1}\right)^{-} \lambda_{1} \text { on } S_{1} \\
L_{2} u_{2}=f \text { in } \Omega_{2}, u_{2}=g \text { on } \Gamma_{2},\left(v \frac{\partial u_{2}}{\partial n}+\left(b_{n}^{2}\right)^{-} u_{2}\right)=\left(b_{n}^{2}\right)^{-} \lambda_{2} \text { on } S_{2} \\
\inf _{\lambda_{1}, \lambda_{2}, v} J_{\alpha}\left(u_{1}, u_{2}, \lambda_{1}, \lambda_{2}, v\right)
\end{gathered}
$$

where $\omega$ and $J_{\alpha}$ have been defined in the previous section.

The variational equations correspondig to (4.3) are

$$
\begin{gathered}
L_{1}^{(0) *} q_{1}=\chi_{12}\left(u_{1}-u_{2}\right) \text { in } \Omega_{1}, \quad\left(b_{n}^{1}\right)^{+} q_{1}=0 \text { on } \partial \Omega_{1} \\
L_{2}^{(0) *} q_{2}=\chi_{12}\left(u_{1}-u_{2}\right) \text { in } \Omega_{2}, \quad q_{2}=0 \text { on } \Gamma_{2} \\
\left(v \frac{\partial q_{2}}{\partial n}+\left(b_{n}^{2}\right)^{+} q_{2}\right)=0 \text { on } S_{2} \\
\alpha\left(b_{n}^{1}\right)^{-} \lambda_{1}+\left(b_{n}^{1}\right)^{-} q_{1}=0 \text { on } S_{1}, \quad \alpha\left(b_{n}^{2}\right)^{-} \lambda_{2}+\left(b_{n}^{2}\right)^{-} q_{2}=0 \text { on } S_{2} \\
\alpha \omega v+\omega q_{1}=0 \text { on } \Omega_{1} .
\end{gathered}
$$

The iterative process that we propose to solve (4.1), (4.2), (4.4) is: for any given $\lambda_{1}^{0}, \lambda_{2}^{0}$,

$$
\begin{gathered}
L_{1} u_{1}^{m}=f+\omega v^{m} \text { in } \Omega_{1}, \quad\left(b_{n}^{1}\right)^{-} u_{1}^{m}=\left(b_{n}^{1}\right)^{-} g \text { on } \Gamma_{1} \\
\left(b_{n}^{1}\right)^{-} u_{1}^{m}=\left(b_{n}^{1}\right)^{-} \lambda_{1}^{m} \text { on } S_{1} \\
L_{2} u_{2}^{m}=f \text { in } \Omega_{2}, \quad u_{2}^{m}=g, \quad\left(v \frac{\partial u_{2}^{m}}{\partial n}+\left(b_{n}^{2}\right)^{-} u_{2}^{m}\right)=\left(b_{n}^{2}\right)^{-} \lambda_{2}^{m} \text { on } S_{2} \\
L_{1}^{(0) *} q_{1}^{m}=\chi_{12}\left(u_{1}^{m}-u_{2}^{m}\right) \text { in } \Omega_{1}, \quad\left(b_{n}^{1}\right)^{+} q_{1}^{m}=0 \text { on } \partial \Omega_{1} \\
L_{2}^{(0) *} q_{2}^{m}=\chi_{12}\left(u_{1}^{m}-u_{2}^{m}\right) \text { in } \Omega_{2}, \quad q_{2}^{m}=0 \text { on } \Gamma_{2} \\
\left(v \frac{\partial q_{2}^{m}}{\partial n}+\left(b_{n}^{2}\right)^{+} q_{2}^{m}\right)=0 \text { on } S_{2} \\
\left(b_{n}^{1}\right)^{-} \lambda_{1}^{m+1}=\left(b_{n}^{1}\right)^{-} \lambda_{1}^{m}-\gamma_{m}\left(\alpha\left(b_{n}^{1}\right)^{-} \lambda_{1}^{m}+\left(b_{n}^{1}\right)^{-} q_{1}^{m}\right) \text { on } S_{1} \\
\left(b_{n}^{2}\right)^{-} \lambda_{2}^{m+1}=\left(b_{n}^{2}\right)^{-} \lambda_{2}^{m}-\gamma_{m}\left(\alpha\left(b_{n}^{2}\right)^{-} q_{2}^{m}+\left(b_{n}^{2}\right)^{-} q_{2}^{m}\right) \text { on } S_{2} \\
\omega v^{m+1}=\omega v^{m}-\gamma_{m}\left(\alpha \omega v^{m}+\omega q_{1}^{m}\right) \text { in } \Omega_{1}, m=0,1, \ldots
\end{gathered}
$$


To study the existence of the solution (when $\alpha=0$, uniqueness for problem (4.1)-(4.3) follows from that of (3.1)-(3.3)) let us consider the adjoint problem

$$
\begin{gathered}
L_{1}^{(0) *} q_{1}=\chi_{12} w \text { in } \Omega_{1}, \quad\left(b_{n}^{1}\right)^{+} q_{1}=0 \text { on } \partial \Omega_{1} \\
L_{2}^{(0) *} q_{2}=\chi_{12} w \text { in } \Omega_{2}, \quad q_{2}=0 \text { on } \Gamma_{2}, \quad\left(v \frac{\partial q_{2}}{\partial n}+\left(b_{n}^{2}\right)^{+} q_{2}\right)=0 \text { on } S_{2}(4.6) \\
\left(b_{n}^{1}\right)^{-} q_{1}=0 \text { on } S_{1}, \quad\left(b_{n}^{2}\right)^{-} q_{2}=0 \text { on } S_{2}, \quad \omega q_{1}=0 \text { in } \Omega_{1} .
\end{gathered}
$$

The latter relation implies that $q_{1}=0$ in $\Omega_{1}$. Therefore, $w=0$ in $\Omega_{12}$. The function $q_{2}$ satisfies the following equations:

$$
\begin{gathered}
L_{2}^{(0) *} q_{2}=0 \text { in } \Omega_{2}, \quad q_{2}=0 \text { on } \Gamma_{2} \\
\left(v \frac{\partial q_{2}}{\partial n}+\left(b_{n}^{2}\right)^{+} q_{2}\right)=0 \text { on } S_{2}, \quad\left(b_{n}^{2}\right)^{-} q_{2}=0 \text { on } S_{2}
\end{gathered}
$$

and (as meas $\left.\left(S_{2}^{-}\right)>0\right)$

$$
L_{2}^{(0) *} q_{2}=0 \text { in } \Omega_{2}, \quad q_{2}=\frac{\partial q_{2}}{\partial n}=0 \text { on } S_{2}^{-} .
$$

Hence, if meas $\left(S_{2}^{-}\right)>0$, then $q_{2}=0$ in $\Omega_{2}$, problem (4.6) has a trivial solution and we conclude that the boundary value problem (3.1)-(3.3) is densely solvable.

Thus, we obtain the assertion.

Proposition 4.1. If meas $\left(S_{2}^{-}\right)>0$, the assertions of Proposition 3.1 hold true for problem (4.1)-(4.3) (instead of (3.1), (3.2), (3.4)) and for process (4.5) (instead of (3.6)).

\section{DOMAIN DECOMPOSITION ALGORITHMS FOR SYSTEMS OF EQUATIONS}

In this section we generalize the above approach to the case of heterogeneous systems of equations. We pay attention to the coefficient $v_{1}$ which can be equal to zero in some subdomain of $\Omega_{1}$ or everywhere in $\Omega_{1}$. In the following we assume that $\bar{\Omega}_{12}=\bigcup_{k=1}^{m} \bar{\Omega}_{12}^{(k)}$, where each subdomain $\Omega_{12}^{(k)}$ is convex or has a smooth boundary $\partial \Omega_{12}^{(k)}$.

5.1. Let us consider the boundary value problem: find $\mathbf{u}_{k}=\left(u_{k, 1}, u_{k, 2}\right), \boldsymbol{\lambda}_{k}=$ 
$\left(\lambda_{k, 1}, \lambda_{k, 2}\right), k=1,2$, and $\mathbf{v}=\left(v_{1}, v_{2}\right)$ such that

$$
\begin{gathered}
-\left(\nabla \cdot\left(v_{1} \nabla\right)\right) \mathbf{u}_{1}+(\nabla \cdot \mathbf{b}) \mathbf{u}_{1}+B_{0} \mathbf{u}_{1}=\mathbf{f}+\omega \mathbf{v} \text { in } \Omega_{1} \\
\left(v_{1} \frac{\partial \mathbf{u}_{1}}{\partial n}+\left(b_{n}^{1}\right)^{-} \mathbf{u}_{1}\right)=\left(b_{n}^{1}\right)^{-} \mathbf{g} \text { on } \Gamma_{1} \\
\left(v_{1} \frac{\partial \mathbf{u}_{1}}{\partial n}+\left(b_{n}^{1}\right)^{-} \mathbf{u}_{1}\right)=\left(b_{n}^{1}\right)^{-} \lambda_{1} \text { on } S_{1} \\
-\left(\nabla \cdot\left(v_{2} \nabla\right)\right) \mathbf{u}_{2}+(\nabla \cdot \mathbf{b}) \mathbf{u}_{2}+B_{0} \mathbf{u}_{2}=\mathbf{f} \text { in } \Omega_{2} \\
\left(v_{2} \frac{\partial \mathbf{u}_{2}}{\partial n}+\left(b_{n}^{2}\right)^{-} \mathbf{u}_{2}\right)=\left(b_{n}^{2}\right)^{-} \boldsymbol{\lambda}_{2} \text { on } S_{2}, \quad \mathbf{u}_{2}=\mathbf{0} \text { on } \Gamma_{2} \\
\mathbf{u}_{1}=\mathbf{u}_{2} \text { in } \Omega_{12}
\end{gathered}
$$

where $\mathbf{b}=\left(b_{1}, b_{2}\right),\left\{\left(b_{n}^{k}\right)^{-}\right\},\left\{\left(b_{n}^{k}\right)^{+}\right\}, \omega$ are defined as in the previous sections, $\mathbf{f}=\left(f_{1}, f_{2}\right), \mathbf{g}=\left(g_{1}, g_{2}\right)$ are given vector functions, $B_{0}=\left\{b_{i j}^{(0)}\right\}$ is a $2 \times 2$ matrix which is assumed to be positive definite (but not necessarily symmetric). We assume that there exist two constants $\mu_{0}, C_{0}>0$ :

$$
\sum_{i, j=1}^{2} b_{i j}^{(0)} \xi_{i} \xi_{j} \geqslant C_{0}|\boldsymbol{\xi}|^{2}, \quad \sum_{i, j=1}^{2} b_{i j}^{(0)} \xi_{i} \xi_{j}+\frac{(\nabla \cdot \mathbf{b})}{2} \sum_{i=1}^{2} \xi_{i}^{2} \geqslant \mu_{0}|\boldsymbol{\xi}|^{2} \quad \forall \boldsymbol{\xi} \in \mathbb{R}^{2} \forall \mathbf{x} \in \bar{\Omega} .
$$

The coefficients $v_{1}, v_{2}$ are bounded and $v_{1} \geqslant 0$ in $\Omega_{1}, v_{2} \geqslant$ const $>0$ in $\Omega_{2}$ and we assume all given data in (5.1) to be smooth.

5.2. To formulate the weak statement of (5.1) we introduce the real Hilbert spaces:

$$
\begin{aligned}
\mathbf{H}_{0, k} \equiv & {\left[L_{2}\left(\Omega_{k}\right)\right]^{2} \equiv \mathbf{H}_{0, k}^{*}, \quad k=1,2, \quad \mathbf{H}_{12} \equiv\left[L_{2}\left(\Omega_{12}\right)\right]^{2} \equiv \mathbf{H}_{12}^{*} } \\
\mathbf{X}_{k}= & \left\{\mathbf{u}_{k}=\left(u_{k, 1}, u_{k, 2}\right): \mathbf{u}_{k} \in \mathbf{H}_{0, k},\right. \\
& \left\|\mathbf{u}_{k}\right\|_{k}=\left(\int_{\Omega_{k}}\left(v_{k}\left|\nabla \mathbf{u}_{k}\right|^{2}+\left|(\mathbf{b} \cdot \nabla) \mathbf{u}_{k}\right|^{2}+\left|\mathbf{u}_{k}\right|^{2}\right) \mathrm{d} \Omega\right. \\
& \left.\left.+\int_{\partial \Omega_{k}}\left(b_{n}^{k}\right)^{+}\left|\mathbf{u}_{k}\right|^{2} \mathrm{~d} \Gamma\right)^{1 / 2}<\infty\right\}, \quad k=1,2 \\
\mathbf{X}_{2}^{(0)}=\left\{\mathbf{u}_{k} \in \mathbf{X}_{2}: \mathbf{u}_{2}=\mathbf{0} \text { on } \Gamma_{2}\right\} & \\
\mathbf{L}_{2}\left(S_{k}^{p}\right)= & \left\{\boldsymbol{\lambda}_{k}:\left\|\boldsymbol{\lambda}_{k}\right\|_{\mathbf{L}_{2}\left(S_{k}^{p}\right)}=\left(\int_{S_{k}}\left(b_{n}^{k}\right)^{p}\left|\boldsymbol{\lambda}_{k}\right|^{2} \mathrm{~d} \Gamma\right)^{1 / 2}<\infty\right\} \\
\mathbf{L}_{2}\left(\Gamma_{k}^{p}\right)= & \left\{\mathbf{g}_{k}:\left\|\mathbf{g}_{k}\right\|_{\mathbf{L}_{2}\left(\Gamma_{k}^{p}\right)}=\left(\int_{\Gamma_{k}}\left(b_{n}^{k}\right)^{p}\left|\mathbf{g}_{k}\right|^{2} \mathrm{~d} \Gamma\right)^{1 / 2}<\infty\right\}, \quad p=-,+, k=1,2 .
\end{aligned}
$$

The spaces $\mathbf{L}_{2}\left(S_{k}^{-}\right), \mathbf{L}_{2}\left(S_{k}^{+}\right), \mathbf{L}_{2}\left(\Gamma_{k}^{-}\right), \mathbf{L}_{2}\left(\Gamma_{k}^{+}\right)$are identified with their dual spaces. Note that we do not identify $\mathbf{X}_{k}$ with $\mathbf{X}_{k}^{*}$. Thus, we have: $\mathbf{X}_{k} \subset \mathbf{H}_{0, k} \equiv \mathbf{H}_{0, k}^{*} \subset \mathbf{X}_{k}^{*}$, 
$k=1,2$. In the sequel, the functions of $\mathbf{H}_{12}$ are extended by zero to include $\Omega_{k} \backslash \Omega_{12}$ and the multiplication of $\mathbf{u} \in \mathbf{H}_{12}$ by $\chi_{12}$ is considered as the trivial continuation of $\mathbf{u}$ to $\Omega_{k} \backslash \Omega_{12}$. We identify $\mathbf{H}_{12}$ with the subspace $(k=1,2)$

$$
\mathbf{H}_{0, k}^{(0)}=\left\{\mathbf{u} \in \mathbf{H}_{0, k}: \mathbf{u} \equiv \mathbf{0} \text { in } \Omega_{k} \backslash \Omega_{12}, \quad\|\mathbf{u}\|_{\mathbf{H}_{0, k}^{(0)}(\Omega)}=\|\mathbf{u}\|_{\mathbf{H}_{12}}=\|\mathbf{u}\|_{\left[L_{2}\left(\Omega_{12}\right)\right]^{2}}\right\} .
$$

To formulate (5.1) in the weak form we multiply the equation in $\Omega_{1}$ by $\widehat{\mathbf{u}}_{1} \in \mathbf{X}_{1}$ in $\mathbf{H}_{0,1}$, the equation in $\Omega_{2}$ by $\widehat{\mathbf{u}}_{2} \in \mathbf{X}_{2}^{(0)}$ in $\mathbf{H}_{0,2}$. After integration by parts we obtain the following problem: find $\mathbf{u}_{1} \in \mathbf{X}_{1}, \mathbf{u}_{2} \in \mathbf{X}_{2}^{(0)}, \lambda_{k} \in \mathbf{L}_{2}\left(S_{k}^{-}\right), k=1,2$, and $\mathbf{v} \in \mathbf{H}_{12}$ such that

$$
\begin{gathered}
a_{1}\left(\mathbf{u}_{1}, \widehat{\mathbf{u}}_{1}\right)=\left(\mathbf{f}, \widehat{\mathbf{u}}_{1}\right)_{\mathbf{H}_{0,1}}+\left(\omega \mathbf{v}, \widehat{\mathbf{u}}_{1}\right)_{\mathbf{H}_{0,1}}+\int_{S_{1}}\left(b_{n}^{1}\right)^{-} \boldsymbol{\lambda}{ }_{1} \cdot \widehat{\mathbf{u}}_{1} \mathrm{~d} \Gamma \\
+\int_{\Gamma_{1}}\left(b_{n}^{1}\right)^{-} \mathbf{g} \cdot \widehat{\mathbf{u}}_{1} \mathrm{~d} \Gamma \quad \forall \widehat{\mathbf{u}}_{1} \in \mathbf{X}_{1}^{(0)} \\
a_{2}\left(\mathbf{u}_{2}, \widehat{\mathbf{u}}_{2}\right)=\left(\mathbf{f}, \widehat{\mathbf{u}}_{2}\right)_{\mathbf{H}_{0,2}}+\int_{S_{2}}\left(b_{n}^{2}\right)^{-} \boldsymbol{\lambda}_{2} \cdot \widehat{\mathbf{u}}_{2} \mathrm{~d} \Gamma \quad \forall \widehat{\mathbf{u}}_{2} \in \mathbf{X}_{2}^{(0)} \\
\chi_{12} \mathbf{u}_{1}=\chi_{12} \mathbf{u}_{2} \text { a.e. in } \Omega_{12}
\end{gathered}
$$

where $\left(\mathbf{u}_{k}, \widehat{\mathbf{u}}_{k}\right)_{\mathbf{H}_{0, k}} \equiv\left(\mathbf{u}_{k}, \widehat{\mathbf{u}}_{k}\right)_{\left[L_{2}\left(\Omega_{k}\right)\right]^{2}}$ and

$$
\begin{aligned}
a_{k}\left(\mathbf{u}_{k}, \widehat{\mathbf{u}}_{k}\right)= & \int_{\Omega_{k}}\left(v_{k} \nabla \mathbf{u}_{k}, \nabla \widehat{\mathbf{u}}_{k}-\mathbf{u}_{k} \cdot(\mathbf{b}, \nabla) \widehat{\mathbf{u}}_{k}+\left(B_{0} \mathbf{u}_{k}\right) \cdot \widehat{\mathbf{u}}_{k}\right) \mathrm{d} \Omega \\
& +\int_{\partial \Omega_{k}}\left(b_{n}^{k}\right)^{+} \mathbf{u}_{k} \cdot \widehat{\mathbf{u}}_{k} \mathrm{~d} \Gamma, \quad k=1,2 .
\end{aligned}
$$

Note that

$$
\int_{\partial \Omega_{2}}\left(b_{n}^{2}\right)^{+} \mathbf{u}_{2} \cdot \widehat{\mathbf{u}}_{2} \mathrm{~d} \Gamma \equiv \int_{S_{2}}\left(b_{n}^{k}\right)^{+} \mathbf{u}_{2} \cdot \widehat{\mathbf{u}}_{2} \mathrm{~d} \Gamma, \quad \mathbf{u}_{2}=\mathbf{0}, \quad \widehat{\mathbf{u}}_{2}=\mathbf{0} \text { on } \Gamma_{2} .
$$

If $v_{1} \equiv 0$ in $\Omega_{1}$,

$$
a_{1}\left(\mathbf{u}_{1}, \widehat{\mathbf{u}}_{1}\right)=\int_{\Omega_{1}}\left(-\mathbf{u}_{1} \cdot(\mathbf{b}, \nabla) \widehat{\mathbf{u}}_{1}+\left(B_{0} \mathbf{u}_{1}\right) \cdot \widehat{\mathbf{u}}_{1}\right) \mathrm{d} \Omega+\int_{\partial \Omega_{1}}\left(b_{n}^{1}\right)^{+} \mathbf{u}_{1} \cdot \widehat{\mathbf{u}}_{1} \mathrm{~d} \Gamma .
$$

If $v_{1}=0$ in $\Omega_{1} \backslash \Omega_{12}$, we have

$$
\begin{aligned}
a_{1}\left(\mathbf{u}_{1}, \widehat{\mathbf{u}}_{1}\right)= & \int_{\Omega_{1} \backslash \Omega_{12}}\left(-\mathbf{u}_{1} \cdot(\mathbf{b}, \nabla) \widehat{\mathbf{u}}_{1}+\left(B_{0} \mathbf{u}\right) \cdot \widehat{\mathbf{u}}_{1}\right) \mathrm{d} \Omega \\
& +\int_{\Omega_{12}}\left(v_{1} \nabla \mathbf{u}_{1} \cdot \nabla \widehat{\mathbf{u}}_{1}-u_{1} \cdot(\mathbf{b}, \nabla) \widehat{\mathbf{u}}_{1}+\left(B_{0} \mathbf{u}\right) \cdot \widehat{\mathbf{u}}_{1}\right) \mathrm{d} \Omega+\int_{\partial \Omega_{1}}\left(b_{n}^{1}\right)^{+} \mathbf{u}_{1} \cdot \widehat{\mathbf{u}}_{1} \mathrm{~d} \Gamma .
\end{aligned}
$$


Let us define the following 'direct' and 'adjoint' operators:

$$
\begin{array}{llll}
L_{1}: \mathbf{X}_{1} \rightarrow \mathbf{X}_{1}^{*}, & \left(L_{1} \mathbf{u}_{1}, \widehat{\mathbf{u}}_{1}\right)_{\mathbf{H}_{0,1}} \equiv a_{1}\left(\mathbf{u}_{1}, \widehat{\mathbf{u}}_{1}\right), & \mathbf{u}_{1} \in \mathbf{X}_{1} & \forall \widehat{\mathbf{u}}_{1} \in \mathbf{X}_{1} \\
L_{2}: \mathbf{X}_{2}^{(0)} \rightarrow \mathbf{X}_{2}^{(0) *}, & \left(L_{2} \mathbf{u}_{2}, \widehat{\mathbf{u}}_{2}\right)_{\mathbf{H}_{0,2}} \equiv a_{2}\left(\mathbf{u}_{2}, \widehat{\mathbf{u}}_{2}\right), & \mathbf{u}_{2} \in \mathbf{X}_{2}^{(0)} & \forall \widehat{\mathbf{u}}_{2} \in \mathbf{X}_{2}^{(0)} \\
L_{1}^{*}: \mathbf{X}_{1} \rightarrow \mathbf{X}_{1}^{*}, & \left(\widehat{\mathbf{u}}_{1}, L_{1}^{*} \mathbf{u}_{1}\right)_{\mathbf{H}_{0,1}} \equiv a_{1}\left(\widehat{\mathbf{u}}_{1}, \mathbf{u}_{1}\right), & \mathbf{u}_{1} \in \mathbf{X}_{1} & \forall \widehat{\mathbf{u}}_{1} \in \mathbf{X}_{1} \\
L_{2}^{*}: \mathbf{X}_{2}^{(0)} \rightarrow \mathbf{X}_{2}^{(0) *}, & \left(\widehat{\mathbf{u}}_{2}, L_{2}^{*} \mathbf{u}_{2}\right)_{\mathbf{X}_{0,2}} \equiv a_{2}\left(\widehat{\mathbf{u}}_{2}, \mathbf{u}_{2}\right), & \mathbf{u}_{2} \in \mathbf{X}_{2}^{(0)} & \forall \mathbf{u}_{2} \in \mathbf{X}_{2}^{(0)} \\
B_{1}: \mathbf{L}_{2}\left(S_{1}^{-}\right) \rightarrow \mathbf{X}_{1}^{*}, & \left(B_{1} \boldsymbol{\lambda}_{1}, \widehat{\mathbf{u}}\right)_{\mathbf{H}_{0,1}} \equiv \int_{S_{1}}\left(b_{n}^{1}\right)^{-} \boldsymbol{\lambda}_{1} \cdot \widehat{\mathbf{u}}_{1} \mathrm{~d} \Gamma, & \boldsymbol{\lambda}_{1} \in \mathbf{L}_{2}\left(S_{1}^{-}\right) & \forall \widehat{\mathbf{u}}_{1} \in \mathbf{X}_{1} \\
B_{2}: \mathbf{L}_{2}\left(S_{2}^{-}\right) \rightarrow \mathbf{X}_{2}^{(0) *}, & \left(B_{2} \boldsymbol{\lambda}_{2}, \widehat{\mathbf{u}}\right)_{\mathbf{H}_{0,2}} \equiv \int_{S_{2}}\left(b_{n}^{2}\right)^{-} \boldsymbol{\lambda}_{2} \cdot \widehat{\mathbf{u}}_{2} \mathrm{~d} \Gamma, & \boldsymbol{\lambda}_{2} \in \mathbf{L}_{2}\left(S_{2}^{-}\right) & \forall \mathbf{u}_{2} \in \mathbf{X}_{2}^{(0)} \\
B_{1, g}: \mathbf{L}_{2}\left(\Gamma_{1}^{-}\right) \rightarrow \mathbf{X}_{1}^{*}, & \left(B_{1, g} \mathbf{g}, \widehat{\mathbf{u}}\right)_{\mathbf{H}_{0,1}} \equiv \int_{\Gamma_{1}}\left(b_{n}^{1}\right)^{-} \mathbf{g} \cdot \widehat{\mathbf{u}}_{1} \mathrm{~d} \Gamma & & \\
& & &
\end{array}
$$

Now problem (5.2) can be rewritten in the operator form as follows:

$$
\begin{gathered}
L_{1} \mathbf{u}_{1}=\mathbf{f}+\omega \mathbf{v}+B_{1} \boldsymbol{\lambda}_{1}+B_{1, g} \mathbf{g} \text { in } \mathbf{X}_{1}^{*} \\
L_{2} \mathbf{u}_{2}=\mathbf{f}+B_{2} \boldsymbol{\lambda}_{2} \text { in } \mathbf{X}_{2}^{(0) *} \\
\lambda_{12} \mathbf{u}_{1}=\chi_{12} \mathbf{u}_{2} \text { a. e. in } \Omega_{12} .
\end{gathered}
$$

Note that the latter relation and the equation $J_{0}\left(\mathbf{u}_{1}, \mathbf{u}_{2}\right) \equiv\left\|\chi_{12}\left(\mathbf{u}_{1}-\mathbf{u}_{2}\right)\right\|_{\left(L_{2}(\Omega)\right)^{2}}^{2} / 2=$ 0 are equivalent.

5.3. Consider the optimal control problem: find $\mathbf{u}_{1} \in \mathbf{X}_{1}, \mathbf{u}_{2} \in \mathbf{X}_{2}^{(0)}, \boldsymbol{\lambda}_{2} \in \mathbf{L}_{2}\left(S_{k}^{-}\right)$, $k=1,2$ and $\mathbf{v} \in \mathbf{H}_{12}$ such that

$$
\begin{gathered}
L_{1} \mathbf{u}_{1}=\mathbf{f}+\omega \mathbf{v}+B_{1} \boldsymbol{\lambda}_{1}+B_{1, g} \mathbf{g} \\
L_{2} \mathbf{u}_{2}=\mathbf{f}+B_{2} \lambda_{2} \\
\inf _{\lambda_{1}, \lambda_{2}, \mathbf{v}} J_{\alpha}\left(\mathbf{u}_{1}, \mathbf{u}_{2}, \boldsymbol{\lambda}_{1}, \boldsymbol{\lambda}_{2}, \mathbf{v}\right)
\end{gathered}
$$

where

$$
\begin{gathered}
J_{\alpha}\left(\mathbf{u}_{1}, \mathbf{u}_{2}, \lambda_{1}, \lambda_{2}, \mathbf{v}\right)=\frac{1}{2}\left(\alpha \int_{S_{1}}\left(b_{n}^{1}\right)^{-}\left|\boldsymbol{\lambda}_{1}\right|^{2} \mathrm{~d} \Gamma+\alpha \int_{S_{2}}\left(b_{n}^{2}\right)^{-}\left|\boldsymbol{\lambda}_{2}\right|^{2} \mathrm{~d} \Gamma\right. \\
\left.+\alpha \int_{\Omega} \omega|\mathbf{v}|^{2} \mathrm{~d} \Gamma+\int_{\Omega} \chi_{12}\left(\mathbf{u}_{1}-\mathbf{u}_{2}\right)^{2} \mathrm{~d} \Omega\right), \quad \alpha=\mathrm{const} \geqslant 0 .
\end{gathered}
$$

If $\alpha=0,(5.4)-(5.6)$ is the weak statement of (5.3). 
The system of variational equations corresponding to (5.4)-(5.6) reads

$$
\begin{aligned}
& a_{1}\left(\mathbf{u}_{1}, \widehat{\mathbf{u}}_{1}\right)=\left(\mathbf{f}, \widehat{\mathbf{u}}_{1}\right)_{\mathbf{H}_{0,1}}+\int_{S_{1}}\left(b_{n}^{1}\right)^{-} \boldsymbol{\lambda}_{1} \cdot \widehat{\mathbf{u}}_{1} \mathrm{~d} \Gamma+\int_{\Gamma_{1}}\left(b_{n}^{1}\right)^{-} \mathbf{g} \cdot \widehat{\mathbf{u}}_{1} \mathrm{~d} \Gamma \quad \forall \widehat{\mathbf{u}}_{1} \in \mathbf{X}_{1} \\
& a_{2}\left(\mathbf{u}_{2}, \widehat{\mathbf{u}}_{2}\right)=\left(\mathbf{f}, \widehat{\mathbf{u}}_{2}\right)_{\mathbf{H}_{0,2}}+\int_{S_{2}}\left(b_{n}^{2}\right)^{-} \boldsymbol{\lambda}_{2} \cdot \widehat{\mathbf{u}}_{2} \mathrm{~d} \Gamma \quad \forall \widehat{\mathbf{u}}_{2} \in \mathbf{X}_{2}^{(0)} \\
& a_{1}\left(\widehat{\mathbf{q}}_{1}, \mathbf{q}_{1}\right)=\left(\chi_{12}\left(\mathbf{u}_{1}-\mathbf{u}_{2}\right), \widehat{\mathbf{q}}_{1}\right)_{\mathbf{H}_{0,1}} \quad \forall \widehat{\mathbf{q}}_{1} \in \mathbf{X}_{1} \\
& a_{2}\left(\widehat{\mathbf{q}}_{2}, \mathbf{q}_{2}\right)=\left(\chi_{12}\left(\mathbf{u}_{1}-\mathbf{u}_{2}\right), \widehat{\mathbf{q}}_{2}\right)_{\mathbf{H}_{0,2}} \quad \forall \widehat{\mathbf{q}}_{2} \in \mathbf{X}_{2}^{(0)} \\
& \alpha\left(b_{n}^{1}\right)^{-} \boldsymbol{\lambda}_{1}+\left(b_{n}^{1}\right)^{-} \mathbf{q}_{1}=\mathbf{0} \quad \text { a. e. on } S_{1} \\
& \alpha\left(b_{n}^{2}\right)^{-} \boldsymbol{\lambda}_{2}+\left(b_{n}^{2}\right)^{-} \mathbf{q}_{2}=\mathbf{0} \quad \text { a. e. on } S_{2} \\
& \alpha \omega \mathbf{v}+\omega \mathbf{q}_{1}=\mathbf{0} \quad \text { a. e. in } \Omega_{1} .
\end{aligned}
$$

Proposition 5.1 [3]. (1) If $v_{1}, v_{2}=$ const in $\Omega_{12}, v_{1} \neq v_{2}$ and one of assumptions (1.4), (1.5) or (1.6) with $\mu \equiv B_{0}+(\operatorname{divb}) \cdot I$ is satisfied, if problem (5.7) for $\alpha=0$ has a solution, this solution is unique.

(2) For any $\alpha>0$, problem (5.7) has the unique solution $\mathbf{u}_{k} \equiv \mathbf{u}_{k}(\alpha), \boldsymbol{\lambda}_{k} \equiv$ $\lambda_{k}(\alpha), k=1,2, \mathbf{v} \equiv \mathbf{v}(\alpha)$.

(3) Problem (5.3) is densely solvable and there holds: $\left\|\chi_{12}\left(\mathbf{u}_{1}(\alpha)-\mathbf{u}_{2}(\alpha)\right)\right\|_{\mathbf{H}_{12}} \rightarrow$ $0, \alpha \rightarrow+0$.

If problem (5.3) has the unique solution $\mathbf{u}_{1}^{(0)}, \mathbf{u}_{2}^{(0)}, \boldsymbol{\lambda}_{1}^{(0)}, \boldsymbol{\lambda}_{2}^{(0)}, \mathbf{v}^{(0)}$, we have $\mathbf{u}_{k}(\alpha) \rightarrow$ $\mathbf{u}_{k}^{(0)}, \boldsymbol{\lambda}_{k}(\alpha) \rightarrow \boldsymbol{\lambda}_{k}^{(0)}, \omega \mathbf{v}(\alpha) \rightarrow \omega \mathbf{v}^{(0)}, k=1,2$.

Let us consider the following iterative process to solve (5.4)-(5.6) approximately: given $\lambda_{1}^{0}, \lambda_{2}^{0}, \mathbf{v}^{0}$, we solve

$$
\begin{aligned}
L_{1} \mathbf{u}_{1}^{m} & =\mathbf{f}+\omega \mathbf{v}^{m}+B_{1} \lambda_{1}^{m}+B_{1, g} \mathbf{g} \\
L_{2} \mathbf{u}_{2}^{m} & =\mathbf{f}+B_{2} \lambda_{2}^{m} \\
\mathbf{z}^{m+1} & =\mathbf{z}^{m}-\gamma_{m} J_{\alpha}^{\prime}\left(\mathbf{u}_{1}^{m}, \mathbf{u}_{2}^{m}, \lambda_{1}^{m}, \lambda_{2}^{m}, \mathbf{v}^{m}\right), \quad m=0,1, \ldots
\end{aligned}
$$

where $\mathbf{z}^{m} \equiv\left(\lambda_{1}^{m}, \lambda_{2}^{m}, \mathbf{v}^{m}\right)$ is the vector of controls and $J_{\alpha}^{\prime}$ is the gradient of $J_{\alpha}$. Using the adjoint equations, the third equation yields

$$
\begin{aligned}
& L_{1}^{*} \mathbf{q}_{1}^{m}=\chi_{12}\left(\mathbf{u}_{1}^{m}-\mathbf{u}_{2}^{m}\right) \\
& L_{2}^{*} \mathbf{q}_{2}^{m}=\chi_{12}\left(\mathbf{u}_{1}^{m}-\mathbf{u}_{2}^{m}\right) \\
& \left(b_{n}^{1}\right)^{-} \lambda_{1}^{m+1}=\left(b_{n}^{1}\right)^{-} \lambda_{1}^{m}-\gamma_{m}\left(\alpha\left(b_{n}^{1}\right)^{-} \boldsymbol{\lambda}_{1}^{m}+\left(b_{n}^{1}\right)^{-} \mathbf{q}_{1}^{m}\right) \quad \text { a. e. on } S_{1} \\
& \left(b_{n}^{2}\right)^{-} \lambda_{2}^{m+1}=\left(b_{n}^{2}\right)^{-} \boldsymbol{\lambda}_{2}^{m}-\gamma_{m}\left(\alpha\left(b_{n}^{2}\right)^{-} \boldsymbol{\lambda}_{2}^{m}+\left(b_{n}^{2}\right)^{-} \mathbf{q}_{2}^{m}\right) \quad \text { a. e. on } S_{2} \\
& \omega \mathbf{v}^{m+1}=\omega \mathbf{v}^{m}-\gamma_{m}\left(\alpha \omega \mathbf{v}^{m}+\omega \mathbf{q}_{1}^{m}\right) \quad \text { a. e. in } \Omega_{1}
\end{aligned}
$$




$$
m=0,1, \ldots
$$

For each $\alpha>0$, one can choose the parameters $\left\{\gamma_{m}\right\}$ can be choosen such that process (5.8) is convergent [11-13].

Theorem 5.1. The following assertions hold true:

(1) For any given $\varepsilon>0$, there exist a small $\alpha>0$ and $M=M(\alpha, \varepsilon)$ such that $\left\|\chi_{12}\left(\mathbf{u}_{1}^{M}-\mathbf{u}_{2}^{M}\right)\right\|_{\mathbf{H}_{12}} \leqslant \varepsilon$, for the solutions of (5.8) ((5.9)); thus

$$
\left\|\chi_{12}\left(\mathbf{u}_{1}^{M}-\mathbf{u}_{2}^{M}\right)\right\|_{\mathbf{H}_{12}} \rightarrow 0 \text { as } \alpha \rightarrow+0, M \rightarrow \infty
$$

and $\mathbf{u}_{1}^{M}, \mathbf{u}_{2}^{M}, \lambda_{1}^{M}, \lambda_{2}^{M}, \mathbf{v}^{M}$ can be considered as an approximate solution of problem (5.3).

(2) If problem (5.3) has the unique solution $\mathbf{u}_{1}^{(0)}, \mathbf{u}_{2}^{(0)}, \lambda_{1}^{(0)}, \lambda_{2}^{(0)}, \mathbf{v}^{(0)}$,

$$
\sum_{k=1}^{2}\left(\left\|\mathbf{u}_{k}^{(0)}-\mathbf{u}_{k}^{M}\right\| \mathbf{x}_{k}+\left\|\lambda_{k}^{(0)}-\lambda_{k}^{M}\right\|_{\mathbf{L}_{2}\left(S_{k}^{-}\right)}\right)+\left\|\omega\left(\mathbf{v}^{(0)}-\mathbf{v}^{M}\right)\right\|_{\mathbf{H}_{12}} \rightarrow 0 \text { as } \alpha \rightarrow+0
$$

$$
M \rightarrow \infty
$$

and relation (5.10) is valid too, where $\mathbf{u}_{1}^{m}, \mathbf{u}_{2}^{m}, \lambda_{1}^{m}, \lambda_{2}^{m}, \mathbf{v}^{m}$ are calculated by the iterative process (5.8).

The proof of this theorem follows from the assertions of Proposition 5.1 and the convergence of iterative process (5.8). If $v_{1} \equiv 0$ in $\Omega_{1}$ or $v_{1}=0$ in $\Omega_{1} \backslash \Omega_{12}$, the same results are still valid.

For simplicity we do not write down algorithm (5.9) ((5.8)) in terms of equations (5.7). This algorithm can be realized numerically in some cases by considering (5.4)-(5.6) as an extremum problem and in form (5.8). In other cases, this algorithm can be considered in form (5.9) as an iterative method to solve system (5.7). In both cases there are specific methods for choosing the parameters $\left\{\gamma_{m}\right\}$ [11-13].

\section{CONCLUSION}

We have analysed some heterogeneous domain decomposition algorithms based on the optimal control approaches to 'overlapping domains $\Omega_{1}, \Omega_{2}$ '. To prove the convergence of algorithms we need to study the uniqueness and existence questions for the boundary value problem and the exact controllability problem 'stated initially'. We proved that to construct approximate solutions, which coincide in $\Omega_{2}=\Omega_{1} \cap \Omega_{2}$ with any accuracy, the 'volume control' must also be introduced into consideration. 'Volume controls' can be introduced into both equations as well.

In [3] we also analyzed the control approach developed in the previous sections to heterogeneous domain decomposition methods in the case of the 'standard' (homogeneous) domain decomposition method for elliptic equations. 
Some of our conclusions are confirmed by numerical experiments described in [4].

We hope that the results presented in this paper will be helpful in the construction of domain decomposition algorithms for other types of equations and boundary conditions, for systems of equations, and for problems in $\Omega \subset \mathbb{R}^{n}, n>2$.

\section{REFERENCES}

1. V. I. Agoshkov, Optimal Control Approaches and Adjoint Equations in the Mathenatical Physics Problem. Inst. of Numer. Math., Russ. Acad. Sci., Moscow, 2003 (in Russian).

2. V. I. Agoshkov, C. Bardos, and S. N. Buleev, Solution of Stokes problem as an inverse problem. Cachan (France): CMLA, ENS, 1999. Preprint No. 9935, 'Computational Methods in Applied Mathematics', 2002, Vol. 2, No. 3, pp. 213-232.

3. V. I. Agoshkov, P. Gervasio, and A. Quarteroni, Optimal control in heterogeneous domain decomposition methods. Tech. Report No. 22/2004 of Seminario Matematico di Brescia, Brescia, Italy, 2004.

4. P. Gervasio, J.-L. Lions, and A. Quarteroni, Heterogeneous coupling by virtual control methods. Numer. Math. (2001) 90, 241-264.

5. S. G. Krein, Linear Equations in Banah Space. Nauka, Moscow, 1971 (in Russian).

6. J.-L. Lions and O. Pironneau, Algorithms paralléles pour la solution de problems aux limites. $C$. R. Acad. Sci., Paris, Sér. I, Math. (1998) 327, 947-952.

7. J.-L. Lions and O. Pironneau, Sur le controle paralléle des systémes distribués. C. R. Acad. Sci., Paris, Sér. I, Math. (1998) 327, 993-998.

8. J.-L. Lions and O. Pironneau, Domain decomposition methods for CAD. C. R. Acad. Sci., Paris, Sér. I, Math. (1999) 328, 73-80.

9. G. I. Marchuk, Methods of Numerical Mathematics. Nauka, Moscow, 1989 (in Russian).

10. A. Quarteroni and A. Valli, Numerical Approximation of Partial Differential Equations. Springer-Verlag, Berlin, 1994.

11. A. N. Tikhonov and V. Ya. Arsenin, Methods for Solving Ill-Posed Problems. Nauka, Moscow, 1974 (in Russian).

12. G. M. Vainikko and A. Yu. Veretennikov, Iterative Procedures in Ill-Posed Problems. Nauka, Moscow, 1974 (in Russian).

13. F. P. Vasiliev, Methods for Solving the Extremum Problems. Nauka, Moscow, 1981 (in Russian). 